\title{
Use of Assistive and Interactive Technology and Relation to Quality of Life in Aging Adults
}

\author{
Sarah Hubner \\ University of Nebraska Omaha \\ shubner@unomaha.edu
}

\author{
Julie Blaskewicz Boron \\ University of Nebraska Omaha \\ jboron@unomaha.edu
}

\author{
Ann Fruhling \\ University of Nebraska Omaha \\ afruhling@unomaha.edu
}

\begin{abstract}
Maintaining quality of life while remaining independent are important goals for aging adults. Recent advancements in technology have provided a variety of physical, cognitive, and social supports to facilitate achieving these goals. This study aimed to improve understanding of older adults' technology needs, preferences, and use to inform development of innovations for this population. Results of this needs assessment revealed that basic technologies are becoming universal, while newer devices are beginning to be adopted; older, well-educated adults may represent early adopters of technology, Further, traditional barriers, including technology complexity and familiarity, may remain obstacles to adoption. This study suggests older adults use technology in ways that support goals and enhance control, autonomy, pleasure, and self-awareness. This research highlights how forthcoming investigations should continue to diversify understanding of technology needs, preferences, and use across sub-populations, and future technology development should utilize this information to tailor services and products to aging consumers.
\end{abstract}

\section{Introduction}

Remaining community-dwelling and autonomous for longer is an important goal for the growing population of aging adults. In support of this goal, assistive and interactive technologies (AITs) can provide a meaningful pathway to bolster independence and enhance quality of life (QOL), especially in later years. AITs are defined as technologies designed to actively assist and promote independence and QOL in populations with functional needs [1]. Aging-specific AITs include low level technologies like walkers and chair lifts and extend to high level innovations such as fall sensors and social robots [2], [3]. Understanding the preferences to use and acceptance of these AITs in target populations is vital to ensuring adoption and facilitating the benefits of innovation [4].
Preference, acceptance, and adoption of AITs are concepts detailed by the technology acceptance model but have been expanded upon since its initial proposal [5], [6]. Briefly, preference suggests a greater liking for one AIT over another, often based on ease of use or usability, while acceptance is a positive or negative perception of an AIT, which can be assessed before or after use [6]. Adoption involves several steps including selecting an AIT, committing to learning or using it, and adopting it for continued use [6].

Barriers to adoption of AITs in aging populations may include a lack of familiarity with, interest in, and access to technology, changing functional ability, and concerns about cost or privacy [2], [4], [7]. More so, function and usability of AITs may drive interest and adoption, while the spectrum of need and circumstance vary widely [2], [4], [7]. Individual characteristics (e.g., age, sex, etc.) create additional variability in needs and attitudes towards technology, while use of technologies can meaningfully impact social and psychological aspects of wellbeing including QOL [2], [4], [8], [9]. Thus, knowing the common needs of older adults, what may be most attractive and beneficial to them in future devices, and how technologies may affect their daily lives, is critical for future understanding AIT impact, effectiveness, and adoption.

Although previous studies have examined AITs for aging adults, the rapid development of increasingly advanced technologies and continually changing familiarity and acceptance of such products warrants regular examination of this knowledge. Additionally, aging adults are notably heterogenous in their preferences and use patterns, highlighting the importance of identifying predictive characteristics for adoption to optimize useful AIT development [4], [9].

The following primary research question was posed: what are the needs, preferences, and patterns of use of AITs in community-dwelling older adults? Subsequently, the research aimed to answer secondary questions including 1) what individual characteristics (e.g., age, gender, etc.) influence technology interest and adoption, and 2) what relationships, if any, exist 
between AIT use, disability, and QOL? These questions were designed to improve understanding of the individual factors which may inform improvements on current and future interventions aimed at QOL and independence for older adults.

The purpose of this research was to contribute to the body of research on aging adults and how their preferences and patterns of AIT adoption vary. Further, understanding older adults' AIT use and preferences may reveal individual characteristics associated with technology adopters, and can contribute to identification of adoption predictors. This could ultimately support design and development of new AITs appropriate to the target populations, meaning that the users would be sufficiently open to, meaningfully interested in, and accepting of novel AITs [4], [7], [10], [11].

To address the research questions and contribute to the field as previously described, a needs assessment was conducted. A questionnaire was designed to assess the relationships between demographic characteristics, functional ability, QOL, and AIT need, adoption, and interest in a population of community-dwelling older adults, aged 60 years and older.

This paper is organized as follows: first, a literature review provides relevant context and theoretical background. This is followed by a discussion of the method of data collection and analysis. Subsequently, the results of the needs assessment are presented. These are discussed and contextualized within the existing data and a theoretical framework. Next, limitations, future directions, and conclusions from the study are explored. Finally, acknowledgements and references are detailed.

\section{Literature Review}

Supporting older adults is a public health priority as the global population continues to age, with the number of adults aged 65 and older projected to more than double by 2050 [12]. Technological innovations are one of the contributors to this demographic shift, facilitating increases in life expectancy [4]. AITs may ultimately be leveraged to reduce strain on public and private support systems, minimizing challenges for aging adults $[4,11]$. By result, there has been a substantial push by health and caregiving communities to encourage integration of assistive technologies in the home environment with goals aimed at enhancing autonomy and QOL for aging adults.

\subsection{Quality of Life}

Throughout social and health research, QOL has been difficult to uniformly define and measure. The relationship between technology use and QOL is of interest to a variety of technology researchers as QOL may be modulated by the integration of technology into various aspects of life, including psychological (e.g., loneliness, stress, activities, cognition) and physical well-being (e.g., health-relevant monitoring/reminders). Previous research has revealed that technology for interactive and communicative purposes increased QOL (measured as subjective well-being) in a sample of aging adults from seven countries [13]. In addition, a cognitive stimulation program delivered via technology was found to be more effective at increasing QOL in aging adults through higher levels of self-esteem, selfefficacy, and autonomy, compared to the program that did not include technology [14]. Informed by this body of previous research, within the current study, QOL was interpreted with respect to its potential effects on psychological (i.e., cognitive and metal health), social and physical wellness, the ability to enjoy normal life activities, and an acceptable standard of health, comfort, and happiness.

\subsection{AITs for Older Adults}

With the increasing volume of AITs available, there are a now a wider variety of suitable devices for aging users. Rapid innovation in AITs has resulted in the development of a broad array of available devices and systems capable of supporting physical, psychological, and social needs of older adults [15]. A variety of AITs are intended for and designed to assist with at least one instrumental activity of daily living (IADL) or activity of daily living (ADL) [2], [16]. Older adults may require support performing IADLs, the daily activities outside of those involved in daily self-care, including driving, shopping, medication, financial management, and housework [17]. A smaller proportion of independentliving adults encounter difficulties with one or more ADLs, associated with basic self-care, including activities such as eating, sleeping, and toileting [17]. IADL and ADL dependence are major determinants of later life disability, loss of independence, and eventual need for assistive and supportive care [17]. AITs may be especially useful for buffering challenges associated with daily physical, cognitive, and social activities, and helping aging adults maintain the independence necessary to remain community dwelling.

Physical AITs provide physical assistance or perform certain functions and include but are not limited to hearing aids, stair lifts, fitness wearables, health and wellness services/management platforms, and mobile apps [18], [19]. Alternatively, cognitive AITs are technologies specifically designed to support cognition, particularly for those with deficiency or decline. These AITs may include home sensor technologies, brain training systems, and medication reminders [20]. Social AITs are typically interactive technologies that help 
provide avenues to socialization, whether with other people or with the technology itself. Social technologies include advanced humanoid, emotional, and companionship social robots (e.g., PARO), as well as lower-level technologies like social and communication platforms, smartphones, texting, and video chat [2],[3].

\subsection{Aging in Place with AITs}

As persons age, physical, psychosocial, and emotional decline/disability may increase the need for additional supports and systems to maintain functional living; this need may be partially or entirely alleviated by AITs [2], [13]. Aging in place, or the tendency to stay in the same community dwelling environment (e.g., private residence or home) as one ages, is highly desired; most aging individuals report a preference for living independently in the community as long as possible [21], [22]. High autonomy, via maintenance of functional ability, is associated with older adults' continued independence [7-8]. Autonomy is globally desirable for aging adults as it can also reduce the costs of care and bolster QOL by maintaining proximity to family and/or support networks [18], [23].

Due to increased average longevity and the desire among older adults to age in place, AITs that can support independent living, especially cost-effective ones, are especially desirable and beneficial [2]. Familiarity, either through exposure or experience, can contribute to preferred use of one AIT over another, also improving attitudes towards and intention to use technologies [24].

\subsection{Theoretical Background}

According to the Theory of Diffusion of Innovations, persons who have previously adopted a technology, or who are quick to adopt, may serve a vital role in continued diffusion of AITs across populations [4], [25]-[27]. In addition, persons may be encouraged to adopt AITs by family and friends who use specific technologies demonstratively or provide support and assistance for learning [18]. Thus, user preferences for AITs (e.g., type and functional use) are important as they drive adoption and the ultimate efficacy of devices. Preferences may be impacted by a variety of factors other than age, including functional ability, assistance needs, comfort with technology, usability, and perceived usefulness.

Persons typically prefer, accept, and adopt AITs they perceive as approachable and useful [4], [18], [25], [26]. Greenhalgh et al. (2004) proposed a model describing adopters of technology, contributing to the previous diffusion theory on the Diffusion of Innovation Model [4], [25]-[28]. These adopters are vital to integrating AITs into a population and are identified by their background (e.g., age, gender, education, needs, skills, etc.) and their perception of an AIT (e.g., use, complexity, etc.). Adoption is negatively associated with age, while adopters are more likely to be educated and of higher income [4]. Previously, males have been found to have greater access to and engagement with technology, although this gender gap appears to be narrowing [4], [29]. Therefore, careful consideration to the purpose of an AIT as well as the cost, range of ability levels, and the intended target population is required. Ease of use and usefulness may be further influenced by design and purpose, and whether the AIT supports a physical, cognitive, or social need [8].

Considering factors which may drive diffusion of innovation, predictive patterns among age, gender, education, and disability statuses have emerged. These factors emphasize the context in which technology is used. This concept is further articulated by the Social Construction of Technology (SCOT) Theory, which serves to highlight the relevance of investigating individual characteristics to elucidate technology use [30], [31]. The SCOT Theory suggests that technology is shaped by social constructs and situations, and that this may in turn inform development and design of AITs [29]-[31]. Ultimately, the individual characteristics which help to predict technology adoption may inform future development.

Contributing to this SCOT theory, a well-known previous study by Czaja et al. (2006) suggested an interconnected structural model between individual characteristics (e.g., age, education), and technology intelligence, perception, and adoption [32]. Results revealed that increased age and lower education negatively predicted technology use [32]. This among other studies has contributed to a growing body of evidence suggesting that older adults are less likely to engage in technology use, while females and persons with higher education are more likely to adopt [4], [10], [29], [32]. Increasing levels of disability also appear to negatively predict technology adoption [33].

\subsection{Current Study}

To summarize, the purpose of this study was to investigate the needs, preferences, and use of AITs in older, community-dwelling adults and to inform current interventions and technology development aimed at enhancing QOL and promoting independence in aging populations. The understanding of acceptance and adoption of AITs in older adults needs to be refreshed and updated as new AITs become available and our society shifts in technology. This is especially vital as use of AITs may support aging goals and improve independence and QOL. 
Variation of adoption across groups is notable; there exists a continued dissonance between newly developed AITs and their acceptance, while existing theories do not appear to fully capture these complex patterns of adoption [4], [7], [10], [11]. Despite their potential utility in older adult populations, actual AIT use may remain low, emphasizing the need for improved understanding of technology adoption [14]. Factors driving diffusion of innovation may help to explain some of the varied patterns of use in aging adults, while characteristics of early adopters may predict AIT use, although these relationships require additional investigation.

Informed by previous research [2], [4], [7]-[11], [34], the investigators hypothesized the following: H1) older adults would preferentially adopt (report at higher frequencies) technologies which they perceived as easyto-use and useful, H2) increasing age, lower education, being male, and greater functional disability would be negatively correlated to technology use; and H3) AIT use would be positively correlated with QOL. The current study assessed the aforementioned relationships via a needs assessment and subsequent descriptive and correlative analyses.

\section{Method}

\subsection{Needs Assessment Development}

A 24-question needs assessment was developed in 2020. The assessment included items related to 1) demographics, 2), difficulties with IADLs/ADLs, 3) knowledge of and experience with using AITs, use of AITs, and overall attitudes towards AITs, 4) QOL, and 5) caregiving (if applicable, 9 additional questions). Only items 1-4 are included in this report, as not all older adults identified as caregivers. This portion of the needs assessment is to be investigated separately. Survey questions included a series of unique inquiries from the authors, as well as questions drawn from existing surveys [2], [35], [36]. The main questionnaire (items 1-4) took older adults approximately 20-30 minutes to complete.

Development of the needs assessment was driven by existing literature previously discussed [2], [4], [7][11], [34]. Briefly, individual characteristics and functional ability notably affect AIT preferences, needs, and use [2]. Despite utility of AITs, a variety of barriers and/or facilitators to adoption may exist, including AIT knowledge and experience, current and previous use, and technology acceptance [2], [4]. Additionally, particularly in aging-oriented fields, a primary goal of AIT adoption includes positively impacting QOL and independence [2], [4]. Unique questions developed by the authors were drawn from this body of knowledge and aimed to assess what AITs were used and what functions on those AITs were utilized.

\subsection{Recruitment and Procedure}

Participants completed the needs assessments online. The self-report survey was administered electronically, in English, via Qualtrics. Due to the ongoing COVID-19 pandemic, no paper-pencil surveys or in-person data were collected. The needs assessment was conducted between March and December of 2020, with the majority of responses accrued between September and December. Completion of the study was voluntary and informed consent was obtained from all subjects before participation. The study protocol was approved by the University of Nebraska Medical Center Institutional Review Board (IRB\# 115-20-EP) prior to participant accrual. Older adults aged 60 and older were eligible for the study. Participants had to be living independently in the community and were required to be fluent in English. Persons residing near Omaha, Nebraska were specifically targeted for inclusion.

Older adult accrual was targeted at $\mathrm{N}=105$. This was based on a power analysis considering two interest groups (older adults and caregivers), with a 95\% confidence level, a $5 \%$ margin of error, and a medium effect size $(d=0.5)$. Participants were recruited through word of mouth, electronic and printed advertisement, and legitimate investigator access. The research team included experts in psychology, gerontology, geriatrics, nursing, assistive technologies for older adults, human computer interaction, and information technology which provided access to a variety of previous research participants and interested parties to aid in accrual. Participant demographics are detailed in the results and Table 1.

\subsection{Measures}

Demographics 3.3.1. Demographic questions were informed by the Behavioral Risk Factor Surveillance System (BRFSS) and the Health and Retirement Study (HRS) [36], [37]. These two nationally representative surveys regularly engage older adults in research and include comprehensive baseline survey questions which are updated frequently to reflect changing response options. Briefly, the BRFSS is an annual survey administered to assess health related risk behaviors as well as disease and chronic health burden [35]. Similarly, the HRS is a longitudinal study conducted regularly to provide multidisciplinary data to elucidate a variety of issues on aging [36]. These surveys provide an effective template for collecting demographic data that would be comparable to other high-quality research databases. 
Functional Ability 3.3.2. IADL/ADL usage was measured using questions based on a study investigating the experience of sharing technology with others and the extent to which older adults interact with and perceive AITs [9]. An additional question associated with technology use and purpose was developed by the authors, based on previous literature [2], [8], [18], [38]. Specifically, the authors included this additional question to expand on technology use by probing utilization of specific, newer technology devices and services (e.g., smart speakers).

Quality of Life 3.3.3. To assess QOL, the Control, Autonomy, Self-Realization, and Pleasure (CASP-19) scale was used. The CASP-19, a summative 19-item scale assessing 4 domains indicated in the name, has been validated and used extensively [39]-[41]. The assessment is specifically recommended for use in aging populations and has been compared in utility to the Life Satisfaction Index [42]. The CASP-19 specifically seeks to investigate one's perception of their 1) control over and within their environment, 2) autonomy from others, 3) personal satisfaction, and 4) awareness of what makes them happy [42]. Items are worded both positively and negatively and are scored on a four-point (0-3) scale ('Often', 'Sometimes', 'Not Often', and 'Never') with 3 equaling the most positive response [42]. This allows for a range of scores 0-57 with higher scores representing a higher QOL [42]. Previous literature has indicated that the CASP-19 score for older adults (aged approximately $65 \pm 10)$ is an average of $42.5(\mathrm{SD}=8.7)$. For adults who are older (aged 75+) or who have little to no education qualifications, scores have been shown to be slightly lower, with an average score of approximately 40.0-40.5 [40], [43]. However, the scale was not originally designed to be understood with cutoffs and is instead interpreted as indicative of relative QOL compared to the study population [40], [44], [45]. This score can then be further broken down and analyzed within the 4 domains and can be utilized correlatively and predictively (in full and in part); additional evidence exists for interpretation of the CASP-19 as a short-form: CASP-12 [40], [44].

\subsection{Statistical Analyses}

All data analysis was done using R v. 4.0.2. Means, standard deviations, and frequency distributions were computed. Significance was set at $\mathrm{p}<0.05$ a priori. Correlational analyses were run to identify multicollinearity in current IADL/ADL ability, future IADL/ADL ability and QOL sections, as well as pairwise correlations among components within all three sections. To investigate relationships between variables of interest and AIT adoption, correlational analyses were conducted between 1) AIT use and demographics, 2) AIT use and AIT function, 3) AIT use and QOL, and 4) AIT use and functional ability.

\section{Results}

A total of 114 older adults completed the online questionnaire. Descriptive statistics revealed that participants were generally female $(74.6 \%)$ and white (94.7\%). The mean age was $70.7(\mathrm{SD}=6.7)$, while the mean CASP-19 (QOL) score was 43.5 (SD=9.4). Of note, the sample represented a highly selective portion of the population; in addition to baseline demographics, most subjects reported high education and moderate average household income.

\section{Table 1. Summary statistics}

\begin{tabular}{lr}
\hline Variable $(\mathrm{N}=114)$ & $\%$ \\
\hline Education & \\
$\quad<$ High School & 0.0 \\
High School or GED & 2.6 \\
Some/2year College/Trade & 13.3 \\
4-Year Degree & 39.5 \\
$\quad$ Graduate/Post Grad & 32.5 \\
Employment & \\
$\quad$ Employed & 23.7 \\
Unemployed & 3.5 \\
Retired & 72.8 \\
Income $(N=98)$ & \\
$<$ \$20,000 & 7.1 \\
\$20,000 - \$34,999 & 13.3 \\
\$35,000 - 49,999 & 16.3 \\
\$50,000 - 74,999 & 26.5 \\
\$75,000 - 99,999 & 16.3 \\
>100,000 & 20.4 \\
Marital Status $(N=112)$ & \\
Single & 6.2 \\
Married & 59.8 \\
Widowed & 16.1 \\
Divorced & 17.9 \\
Separated & 0.0 \\
\hline
\end{tabular}

Regarding IADL/ADL status, respondents were in generally good health with low disability. Considering 10 of 11 possible IADL/ADL questions assessing subjective disability/difficulty (with a minimum possible score of 10 (low disability), and maximum possible score of 30 (high disability)), the average score for present ability was $12.43(\mathrm{SD}=0.26)$. The average for expected future ability was $13.02(\mathrm{SD}=0.22)$. One question related to public transportation was removed from these averages as most participants indicated that the activity was "not applicable" to them. 
Use of AITs was quantified as a binary Yes/No, with participants simply selecting the technologies that they used from a list. Participants generally reported a high use of basic AITs (smartphones, computers, laptops, and tablets) for any purpose (Table 2). Regarding function, these basic AITs were favorably used for email (99.1\%), internet $(97.4 \%)$, texting $(92.1 \%)$, directions/navigation $(91.2 \%)$, and pictures $(90.4 \%)$ (Table 3). There was greater diversity in use of newer AITs (e.g., smart speakers $(44.7 \%)$ versus smart home technology $(18.4 \%)$ ); a high proportion of respondents $(68.4 \%)$ reported using telehealth technologies (Table 3).

Table 2. Proportion of subjects who use AITs

\begin{tabular}{ll}
\hline Technology & \% Yes \\
\hline Internet & 99.1 \\
Smartphone & 90.4 \\
Computer/Laptop & 87.7 \\
Tablet & 71.1 \\
Telehealth Technology (e.g., OneChart) & 68.4 \\
Smart TV Technology & 55.3 \\
Smart Speaker Technology & 44.7 \\
Grocery Services (e.g., delivery) & 27.2 \\
Transportation Services (e.g., Uber) & 22.8 \\
Lodging Services (e.g., AirBnB) & 20.2 \\
Smart Home Technology (e.g., Ring) & 18.4 \\
\hline
\end{tabular}

Note: "Use" assessed as binary Yes/No

\section{Table 3. Proportion of subjects who use smartphone, computer, laptop, or tablet for listed purpose}

\begin{tabular}{ll}
\hline Technology Purpose & \% Yes \\
\hline Email & 99.1 \\
Internet & 97.4 \\
Texting & 92.1 \\
Directions/Navigation & 91.2 \\
Pictures & 90.4 \\
Telephone Calls & 86.8 \\
Social Media & 81.6 \\
Books & 72.8 \\
Video Calls & 71.1 \\
Videos/Music & 63.2 \\
Managing Schedules & 57.9 \\
Games & 57.9 \\
Do Not Use for Above Purposes & 11.4 \\
\hline
\end{tabular}

Note: "Use" assessed as binary Yes/No

No significant relationships were identified within correlational analyses between AIT use and demographics nor AIT use and functional ability.
Correlations between AIT use and AIT function revealed that smartphone use was positively correlated with texting $(\rho=0.79, \mathrm{p}<.001)$ and email $(\rho=0.29$, $\mathrm{p}<.001$ ), in addition to less-frequently reported activities like pictures $(\rho=0.40, \mathrm{p}<.001)$ and social media $(\rho=0.30, \mathrm{p}<.001)$. Smartphones were also associated with telephone calls $(\rho=0.66, \mathrm{p}<.001)$, suggesting use of this technology may be ubiquitous. No other significant relationships were identified.

Considering correlational analyses between AIT use and QOL (CASP-19 score), results revealed that smart speaker use and QOL were positively correlated $(\rho=0.32, \mathrm{p}<.001)$. Smart speakers included the list items 1) Alexa/Amazon Echo, 2) Google Home, Google Dot, Google Mini, or Google Nest, 3) Apple Home Pod, and 4) Other Smart/Voice Controlled Speaker. No other significant relationships were identified.

\section{Discussion}

The aim of this research was to investigate relationships between individual characteristics, technology and QOL. The purpose of this study was to assess the needs, preferences, and use of AITs in older adults to improve future innovation and research. Results are discussed within the context of our hypotheses.

H1) Within this large, albeit relatively homogenous, sample, use of basic technologies (e.g., communication AITs) was nearly universal, while some newer technologies (e.g., health, entertainment, smart home AITs etc.) were beginning to be adopted. Considering the Diffusion of Innovation Model discussed previously [25], [27], it may be that irrelevant of background or individual characteristics, persons favorably adopt communication and information technologies (e.g., smartphones, internet) as they meet important AIT attributes for acceptance (e.g., advantage, complexity). This is relevant as the adoption of AITs and their social context drives development and defines use, per the SCOT theory [30], [31]. Further, previous research suggests that if technology is sufficiently beneficial, the positive outcomes of adoption can outweigh any concerns or problems held by the user [10]. This may suggest that innovations for older adults could retain some level of complexity or cost, but only for AITs with the highest levels of benefit. Otherwise, when targeting aging populations, it is necessary to evaluate the cost versus benefit of use and ensure a positive balance, in order to encourage high rates of adoption and promote a better user experience. Considering the intersection of both Diffusion of Innovation and SCOT theories, it is important to understand the context in which a technology is used 
and the likely adopter characteristics to drive technology utilization.

H2) Within this population, no specific correlations between demographics and AIT use were identified. However, our study population was well educated with a higher income. Additionally, the sample was overwhelmingly white, healthy, and predominantly female. High telehealth utilization despite low current and expected disability status, combined with high education levels, may suggest better health literacy, although this concept was not specifically measured. While these demographic factors may skew correlational results, it may also support that persons with higher education and more economic resources tend to adopt technology more willingly, extending to participation in online, technology-based research. Similarly, these resources may have related to this population of females adopting AITs more favorably than males; however, females disproportionately participated in this study and generally engage in research more readily overall [46]. Nonetheless, these findings, while qualitative, may provide insight for future study designs as they emphasize the importance of targeting a variety of underrepresented research populations including older adults, non-white populations, and persons with lower education, economic resources, and technology access.

H3) Considering QOL, results of this study suggests that in well-educated, low-disability adults, use of newer technologies (e.g., smart speakers) may be positively correlated with QOL, although directionality $\mathrm{f}$ these relationships could not be assessed. The average QOL in the study sample was comparable to prior research on older adults and scores are in line with other similarly well-educated, older samples [26], [29]. However, due to participant homogeneity, it is less appropriate to compare participants' QOL across age, education, and income levels. Instead, it is more meaningful to discuss QOL and its relationship to AITs within the domains of the CASP-19 scale itself: control, autonomy, pleasure, and self-awareness [39], [42].

Considering the CASP-19 concept of control, respondents displayed authority over and within their environments as evidenced by their current and future IADL/ADL statuses, which suggested that they were in relatively good health with low subjective disability. Additionally, they demonstrated notable autonomy from others, as this study only included community-dwelling adults. Further, the sense of control and autonomy within this sample may be emphasized by the nearuniversal use of basic technologies, indicative of flexibility and adaptiveness as well as acceptance of helpful devices to aid in daily activities. It may be that this use of AITs bolsters QOL, or that QOL increases adaptability and openness to AIT adoption; further investigation is warranted.

The high use of AITs within this sample population suggests that this group of aging adults was comfortable with and interested in using basic smart phone technology, including functions like texting (92.1\%) and email (99.1\%), and that they were branching out to other technologies (e.g., smart speakers, 44.7\%). The AITs endorsed by the aging adults in our sample could be used for a wide range of communicative and personal assistance activities, such as social communications with family/friends, keeping up with appointments, reminders, etc. Further, these persons with high technology use might be described as early adopters of technology [25]. Considering the CASP-19 aspects of pleasure and self-awareness, these early adopters of AITs may be better able to maintain QOL and functional ability through technology use. Alternatively, persons with high QOL and/or ability may be more likely to be early adopters of technology. This reiterates the call for continued investigation.

The discrepancy in use between older, more basic technologies within this sample and newer, 'highertech' systems may emphasize the potential barriers to use, low perceived usefulness, and varied adoption patterns in older adult samples, supported by extensive previous research [2], [8], [18], [19], [47], [48]. Further, it may be that other factors or spurious variables are partially or entirely responsible for the relationships identified within this study. Ultimately, continued research on AIT needs, preferences, and acceptance is required.

\section{Limitations}

Methodological challenges of investigating aging adults' technology usage should be acknowledged. The sample from the current study is selective. As data were collected during the ongoing COVID-19 pandemic, all responses had to be gathered via an online survey in order to prioritize participant safety. This requirement may have excluded people that did not have regular internet access and/or were not comfortable responding to a survey online. It was challenging to gain sufficient responses, which suggests that simply utilizing an online survey may not be sufficient to attract a generalizable older adult sample. Future research, with other samples of aging adults will need to employ a wide range of responding options to collect data, which may include online surveys, hard copy completion, in-person administration, and/or facilitation of completion via an interview either in-person or over the phone. 


\section{Future Research}

Findings suggested that future research should aim to diversify understanding of needs and preferences across sub-groups of aging adults, beyond early adopters of technology. Additionally, ongoing investigations should seek to better understand use patterns and the potential utility of AITs in other samples, such as caregivers, persons with dementia, and older adults in rural areas where connectivity may not be constant. Each of these subsets of the aging population could benefit from AITs, however, their preferred technology and use frequency may differ. Greater understanding of needs and preferences within a broader range of aging populations is ultimately ideal for suggesting technology integration to offer support, promote autonomy, and bolster independence. Moreover, technology innovation and development should continually focus on devices and services that are approachable, affordable, and accessible for populations who are not readily interested in adoption, while continuing to attract early adopters with newer devices and services.

\section{Conclusions}

The purpose of this study was to investigate the use of AITs among older adults and to understand their relationship to QOL. Results suggest that basic technologies are becoming universal, while newer AITs are beginning to be adopted. Further, this study aligns with previous research including the theory of Diffusion of Innovation, such that adopters of technology may engage with AITs based on the innovation's benefit and their own perception of its attributes. Further, individual characteristics may drive early adopters of technology, while QOL may be associated with AIT use. Understanding how aging adults currently use AITs to improve their QOL and support independence is helpful as we look to development of future technologies for older persons. Important areas to concentrate future integration of AITs may include safety, health (e.g., nutrition, blood pressure, activity level, fall detection, etc.), communication (e.g., for those with hearing or visual impairments), and additional IADL assistance (e.g., reminders, scheduling, etc.). These AIT advancements will help support aging adults, caregivers, and adults with specific needs, such as those with chronic or progressive diseases such as dementia. As maintaining independence is a goal for aging adults and caregivers, it is essential to prioritize innovation and use of AITs that people are comfortable with using, which also have the potential to increase QOL, functional ability, and independence.

\section{Acknowledgements}

This study was supported by a Nebraska Collaboration Initiative Planning Grant and was a joint effort between researchers at the University of Nebraska Omaha and University of Nebraska Medical Center. The authors would like to thank Dr. Natalie Manley, Dr. Marcia Shade, Meaghan Walls, and Joshua Idachaba for their contributions to this study.

\section{References}

[1] Technology-Related Assistance for Individuals with Disabilities Act. 1988. [Online]. Available: https://www.congress.gov/bill/100th-congress/senatebill/2561

[2] J. Miller, C. Ward, C. Lee, L. D'Ambrosio, and J. Coughlin, "Sharing is caring: The potential of the sharing economy to support aging in place," Gerontol. Geriatr. Educ., pp. 1-23, Jan. 2018, doi: 10.1080/02701960.2018.1428575.

[3] S. A. McGlynn, S. Kemple, T. L. Mitzner, C.-H. A. King, and W. A. Rogers, "Understanding the potential of PARO for healthy older adults," Int. J. Hum.Comput. Stud., vol. 100, pp. 33-47, Apr. 2017, doi: 10.1016/j.ijhcs.2016.12.004.

[4] T. L. Mitzner et al., "Technology Adoption by Older Adults: Findings From the PRISM Trial," The Gerontologist, vol. 59, no. 1, pp. 34-44, Jan. 2019, doi: 10.1093/geront/gny113.

[5] K. Renaud and J. van Biljon, "Predicting technology acceptance and adoption by the elderly: a qualitative study," in Proceedings of the 2008 annual research conference of the South African Institute of Computer Scientists and Information Technologists on IT research in developing countries: riding the wave of technology, New York, NY, USA, Oct. 2008, pp. 210-219. doi: 10.1145/1456659.1456684.

[6] F. D. Davis, "Perceived Usefulness, Perceived Ease of Use, and User Acceptance of Information Technology," MIS Q., vol. 13, no. 3, pp. 319-340, 1989, doi: 10.2307/249008.

[7] K. Chen and V. W. Q. Lou, "Measuring Senior Technology Acceptance: Development of a Brief, 14Item Scale," Innov. Aging, vol. 4, no. 3, May 2020, doi: 10.1093/geroni/igaa016.

[8] S. T. M. Peek et al., "Older Adults' Reasons for Using Technology while Aging in Place," Gerontology, vol. 62 , no. 2, pp. 226-237, 2016, doi: $10.1159 / 000430949$.

[9] L. C. van Boekel, S. T. Peek, and K. G. Luijkx, "Diversity in Older Adults' Use of the Internet: Identifying Subgroups Through Latent Class Analysis," J. Med. Internet Res., vol. 19, no. 5, p. e6853, May 2017, doi: 10.2196/jmir.6853. 
[10] A. Tsertsidis, E. Kolkowska, and K. Hedström, "Factors influencing seniors' acceptance of technology for ageing in place in the post-implementation stage: A literature review," Int. J. Med. Inf., vol. 129, pp. 324 333, Sep. 2019, doi: 10.1016/j.ijmedinf.2019.06.027.

[11] W. K. Bong, A. Bergland, and W. Chen, "Technology Acceptance and Quality of Life among Older People Using a TUI Application," Int. J. Environ. Res. Public. Health, vol. 16, no. 23, p. 4706, Dec. 2019, doi: 10.3390/ijerph16234706.

[12] United Nations, Department of Economic and Social Affairs, Population Division, "World Population Ageing 2019: Highlights.," ST/ESA/SER.A/430, 2019. [Online]. Available:

https://www.un.org/en/development/desa/population/p ublications/pdf/ageing/WorldPopulationAgeing2019Highlights.pdf

[13] G. Nimrod, "Aging Well in the Digital Age: Technology in Processes of Selective Optimization with Compensation," J. Gerontol. Ser. B, vol. 75, no. 9, pp. 2008-2017, Oct. 2020, doi: 10.1093/geronb/gbz111.

[14] D. Cebreros-Valenzuela et al., "Efficacy of a cognitive stimulation programme using technology on older adults' self-esteem, self-efficacy, and autonomy," Rev. Latinoam. Psicol., vol. 52, pp. 42-50, Jun. 2020, doi: 10.14349/rlp.2020.v52.5.

[15] D. A. Lindeman, K. K. Kim, C. Gladstone, and E. C. Apesoa-Varano, "Technology and Caregiving: Emerging Interventions and Directions for Research," The Gerontologist, vol. 60, no. Suppl 1, pp. S41-S49, Feb. 2020, doi: 10.1093/geront/gnz178.

[16] F. G. Miskelly, "Assistive technology in elderly care," Age Ageing, vol. 30, no. 6, pp. 455-458, Nov. 2001, doi: 10.1093/ageing/30.6.455.

[17] J. M. Carmona-Torres, M. A. Rodríguez-Borrego, J. A. Laredo-Aguilera, P. J. López-Soto, E. SantacruzSalas, and A. I. Cobo-Cuenca, "Disability for basic and instrumental activities of daily living in older individuals," PLOS ONE, vol. 14, no. 7, p. e0220157, Jul. 2019, doi: 10.1371/journal.pone.0220157.

[18] K. Luijkx, S. Peek, and E. Wouters, “'Grandma, You Should Do It - It's Cool' Older Adults and the Role of Family Members in Their Acceptance of Technology," Int. J. Environ. Res. Public. Health, vol. 12, no. 12 , pp. 15470-15485, Dec. 2015, doi: 10.3390/ijerph121214999.

[19] E. Tural, D. Lu, and D. A. Cole, "Factors predicting older Adults' attitudes toward and intentions to use stair mobility assistive designs at home," Prev. Med. Rep., vol. 18, p. 101082, Jun. 2020, doi: 10.1016/j.pmedr.2020.101082.

[20] A. Mitseva et al., "Gerontechnology: Providing a Helping Hand When Caring for Cognitively Impaired Older Adults-Intermediate Results from a Controlled Study on the Satisfaction and Acceptance of Informal Caregivers," Curr. Gerontol. Geriatr. Res., vol. 2012, 2012, doi: 10.1155/2012/401705.
[21] H. Matsumoto, T. Naruse, M. Sakai, and S. Nagata, "Who prefers to age in place? Cross-sectional survey of middle-aged people in Japan," Geriatr. Gerontol. Int., vol. 16, no. 5, pp. 631-637, May 2016, doi: 10.1111/ggi.12503.

[22] J. Binette and K. Vasold, "2018 Home and Community Preferences: A National Survey of Adults Ages 18Plus," Washington, DC: AARP Research, Aug. 2018. https://doi.org/10.26419/res.00231.001 (accessed Jun. 05, 2021).

[23] M. Illario, M. M. R. Vollenbroek-Hutten, D. W. Molloy, E. Menditto, G. Iaccarino, and P. Eklund, "Active and Healthy Ageing and Independent Living 2016," J. Aging Res., vol. 2016, 2016, doi: $10.1155 / 2016 / 8062079$.

[24] E. Vaportzis, M. Giatsi Clausen, and A. J. Gow, "Older Adults Experiences of Learning to Use Tablet Computers: A Mixed Methods Study," Front. Psychol., vol. 9, Sep. 2018, doi: 10.3389/fpsyg.2018.01631.

[25] T. Greenhalgh, G. Robert, F. Macfarlane, P. Bate, and O. Kyriakidou, "Diffusion of Innovations in Service Organizations: Systematic Review and Recommendations," Milbank Q., vol. 82, no. 4, pp. 581-629, 2004, doi: 10.1111/j.0887378X.2004.00325.X.

[26] E. S. McConnell et al., "Diffusion of Innovation in Long-Term Care (DOI-LTC) Measurement Battery," Res. Gerontol. Nurs., vol. 5, no. 1, pp. 64-76, Jan. 2012, doi: 10.3928/19404921-20110602-04.

[27] E. M. Rogers, Diffusion of Innovations, 4th Edition, 4th ed. New York, NY: The Free Press, 1995.

[28] X. Zhang, P. Yu, J. Yan, and I. Ton A M Spil, "Using diffusion of innovation theory to understand the factors impacting patient acceptance and use of consumer e-health innovations: a case study in a primary care clinic," BMC Health Serv. Res., vol. 15, p. 71, Feb. 2015, doi: 10.1186/s12913-015-0726-2.

[29] L. J. Dixon et al., "Gendered Space: The Digital Divide between Male and Female Users in Internet Public Access Sites," J. Comput.-Mediat. Commun., vol. 19, no. 4, pp. 991-1009, 2014, doi: 10.1111/jcc4.12088.

[30] E. Wheeler, "The Social Shaping of Technology edited by Donald MacKenzie and Judy Wajcman (Open University Press, Milton Keynesand Philadelphia, 1985) pp. vii + 237, ISBN 0-335-15027-6,"

Prometheus, vol. 3, no. 2, pp. 466-469, Dec. 1985, doi: 10.1080/08109028508629013.

[31] T. J. Pinch and W. E. Bijker, "The Social Construction of Facts and Artefacts: or How the Sociology of Science and the Sociology of Technology might Benefit Each Other," Soc. Stud. Sci., vol. 14, no. 3, pp. 399-441, Aug. 1984, doi: 10.1177/030631284014003004.

[32] S. J. Czaja et al., "Factors Predicting the Use of Technology: Findings From the Center for Research and Education on Aging and Technology Enhancement (CREATE)," Psychol. Aging, vol. 21, no. 2, pp. 333-352, Jun. 2006, doi: 10.1037/08827974.21.2.333. 
[33] N. M. Gell, D. E. Rosenberg, G. Demiris, A. Z. LaCroix, and K. V. Patel, "Patterns of technology use among older adults with and without disabilities," The Gerontologist, vol. 55, no. 3, pp. 412-421, Jun. 2015, doi: 10.1093/geront/gnt166.

[34] L. Liu, E. Stroulia, I. Nikolaidis, A. Miguel-Cruz, and A. Rios Rincon, "Smart homes and home health monitoring technologies for older adults: A systematic review," Int. J. Med. Inf., vol. 91, pp. 44-59, Jul. 2016, doi: 10.1016/j.ijmedinf.2016.04.007.

[35] Centers for Disease Control, "2019 BRFSS Questionnaire." U.S. Department of Health and Human Services, National Center for Chronic Disease Prevention and Health Promotion, Division of Population Health, 2021. [Online]. Available: https://www.cdc.gov/brfss/questionnaires/index.htm

[36] Health and Retirement Study, "Health and Retirement Study, 2018 Cross Wave Tracker.” Institute for Social Research, University of Michigan, Regents of the University of Michigan, 2020. [Online]. Available: https://hrsdata.isr.umich.edu/data-products/crosswave-tracker-file

[37] Centers for Disease Control (CDC), "Behavioral Risk Factor Surveillance System Survey Data," U.S. Department of Health and Human Services, Centers for Disease Control and Prevention, 2019, Atlanta, Georgia, Dec. 2019. Accessed: Jan. 28, 2020. [Online]. Available: https://www.cdc.gov/brfss/questionnaires/index.htm

[38] R. J. Holden and B.-T. Karsh, "The technology acceptance model: its past and its future in health care," J. Biomed. Inform., vol. 43, no. 1, pp. 159-172, Feb. 2010, doi: 10.1016/j.jbi.2009.07.002.

[39] M. Hyde, R. D. Wiggins, P. Higgs, and D. B. Blane, "A measure of quality of life in early old age: The theory, development and properties of a needs satisfaction model (CASP-19)," Aging Ment. Health, vol. 7, no. 3, pp. 186-194, May 2003, doi: 10.1080/1360786031000101157.

[40] C. Borrat-Besson, V.-A. Ryser, and J. Gonçalves, "An evaluation of the CASP-12 scale used in the Survey of Ageing and Retirement in Europe (SHARE) to measure Quality of Life among people aged 50+," Lausanne, Switzerland: FORS, paper 2015-4, 2015. Accessed: Jun. 08, 2021. [Online]. Available: /paper/An-evaluation-of-the-CASP-12-scale-used-inthe-of-

Ryser/fa0b8f8bb6af4cf269e70a788ce18a5643989291
[41] F. Lima et al., "Quality of Life amongst Older Brazilians: A Cross-Cultural Validation of the CASP19 into Brazilian-Portuguese," 9 E94289, doi: doi:10.1371/journal.pone.0094289.

[42] P. Higgs, M. Hyde, R. Wiggins, and D. Blane, "Researching Quality of Life in Early Old Age: The Importance of the Sociological Dimension," Soc. Policy Adm., vol. 37, no. 3, pp. 239-252, 2003, doi: 10.1111/1467-9515.00336.

[43] G. Netuveli, R. Wiggins, Z. Hildon, S. Montgomery, and D. Blane, "Quality of life at older ages: evidence from the English longitudinal study of aging (wave 1)," J. Epidemiol. Community Health, no. 60, pp. 357363, 2006, doi: 10.1136/jech.2005.040071.

[44] D. Howel, "Interpreting and evaluating the CASP-19 quality of life measure in older people," Age Ageing, vol. 41, no. 5, pp. 612-617, Sep. 2012, doi: 10.1093/ageing/afs023.

[45] J. Sim, B. Bartlam, and M. Bernard, "The CASP-19 as a measure of quality of life in old age: evaluation of its use in a retirement community," Qual. Life Res., vol. 20, pp. 997-1004, 2001, doi: https://doi.org/10.1007/s11136-010-9835-x.

[46] D. C. Glass et al., "A telephone survey of factors affecting willingness to participate in health research surveys," BMC Public Health, vol. 15, p. 1017, Oct. 2015, doi: 10.1186/s12889-015-2350-9.

[47] S. M. Golant, "A theoretical model to explain the smart technology adoption behaviors of elder consumers (Elderadopt)," J. Aging Stud., vol. 42, pp. 56-73, Aug. 2017, doi: 10.1016/j.jaging.2017.07.003.

[48] D. Yagil, M. Cohen, and J. D. Beer, “Older Adults' Coping With the Stress Involved in the Use of Everyday Technologies," J. Appl. Gerontol., vol. 35, no. 2, pp. 131-149, Feb. 2016, doi: $10.1177 / 0733464813515089$. 